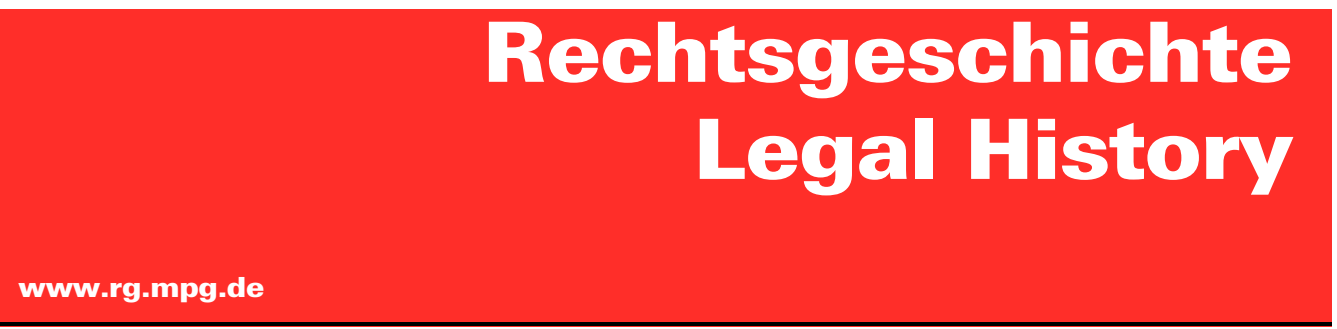

http://www.rg-rechtsgeschichte.de/rg26

$\operatorname{Rg} 26_{2018}$

$498-507$

Zitiervorschlag: Rechtsgeschichte - Legal History Rg 26 (2018)

http://dx.doi.org/10.12946/rg26/498-507

\title{
Holger Knudsen*
}

\section{Die englische Kolonialgesetzgebung für Helgoland - »Ordinances of Heligoland «}

Zugleich eine kurze Besprechung des Buches »Helgoland« von Jan Rüger

[The English Colonial Law-Making for Heligoland - »Ordinances of Heligoland «. Including a Review of the Book »Helgoland« by Jan Rüger]

* Max-Planck-Institut für ausländisches und internationales Privatrecht, Hamburg (Emeritus) / Hochschule für Technik, Wirtschaft und Kultur Leipzig (Emeritus), holger_knudsen@yahoo.de 


\section{Abstract}

The contribution deals with a widely unknown and formerly inaccessible part of English colonial law-making: the small body of legal rules for the colony of Heligoland (1807-1890). Even though Heligoland was a very small and rather insignificant colony, the ordinances are interesting for three reasons: the indulgent treatment of the local population (given that Heligoland was one of the very few European colonies of the empire), the geographical vicinity of German-speaking territories, and in particular, the emerging German Empire after 1871. All of these are reflected in the application of foreign (German) laws (strand laws, monetary laws, metric rules) to the colony. This made Heligoland a unique case compared to other colonies.

The contribution provides an overview of the pre-history of the introduction of the ordinances (1807-1863), and it explains the why, whence and whither of the 76 ordinances that were passed between 1864 and 1889. It is completed by a chronological list of the ordinances.

Keywords: English colonial law-making, Governor Maxse, ordinances, Heligoland, Anglo-German treaty 
Holger Knudsen

\title{
Die englische Kolonialgesetzgebung für Helgoland - »Ordinances of Heligoland «
}

\author{
Zugleich eine kurze Besprechung des Buches »Helgoland« von Jan Rüger ${ }^{1}$
}

Habent sua fata libelli

Im Jahr 2001 reiste ich, damals noch Direktor der Bibliothek des Max-Planck-Instituts für ausländisches und internationales Privatrecht in Hamburg, nach London, um mit meinem Kollegen Jules Winterton, Direktor der Bibliothek des Institute of Advanced Legal Studies (IALS), den (erfolgreich abgeschlossenen) Austausch besonders wertvoller Dubletten und die gegenseitige Ergänzung des lückenhaften Bestandes von afrikanischen Gesetzblättern zu besprechen. Mir wurde irrtümlicherweise ein sehr unscheinbarer Band vorgelegt, der sich bei näherem Hinsehen nicht als Doppelexemplar, sondern als einzigartiges Unikat erwies: die komplette Sammlung der englischen Kolonialgesetzgebung (mit den zugehörigen handschriftlichen Entwürfen aus dem Sekretariat der jeweiligen Gouverneure) für die Insel Helgoland aus den vom IALS übernommenen Beständen der aufgelösten Bibliothek des Foreign and Commonwealth Office.

Der Band verblieb vereinbarungsgemäß in London, er wurde aber fairerweise von den dortigen Kollegen vollständig gescannt und frei zugänglich ins Internet gestellt, ${ }^{2}$ ist aber natürlich auch in der Bibliothek des Institute of Advanced Legal Studies einsehbar. ${ }^{3}$ Das Interesse war geweckt und so sammelte ich im Folgejahr zusammen mit meiner damaligen Assistentin Dr. Michéle John ${ }^{4}$ Materialien für einen Aufsatz über die Ordinances. Dass das Projekt trotz gelegentlicher späterer dis- kreter Nachfragen von Frau John dann völlig liegenblieb, ist absolut unverzeihlich und nur mit anderweitiger Arbeitsbelastung zu erklären. Das Buch von Rüger war nun der Anlass, sich mit Hilfe der 2001/02 gesammelten Materialien des Themas erneut anzunehmen.

\section{Helgoland - Deutschland, England und ein Felsen in der Nordsee}

Der Autor, Professor für Geschichte am Birkbeck College der University of London, »behandelt Helgoland als Spiegelbild und Mikrokosmos einer großen europäischen Geschichte. Er zeigt die Insel jenseits der Nationen und Völker als einen Ort der historischen Vielfalt und als Mahnmal für einen dauerhaften europäischen Frieden ${ }^{5}{ }^{5}$

Die Übersetzung ist sorgfältig, gut gelungen und sehr gut lesbar. An keiner Stelle entsteht das bei (schlechten) Übersetzungen sonst recht häufige Gefühl, man könne den Text problemlos ins Original zurückübersetzen. Störend ist lediglich die englische Benennung der dänischen Könige und schleswig-holsteinischen Herzöge als »Frederick« statt des im deutschen Sprachgebrauch üblichen Frederik oder Friedrich.

Rüger beschreibt die deutsch-englische Geschichte mit ihren Verwebungen und Verwerfungen am Beispiel und aus der Sicht ausgesuchter Akteure und häufig anekdotisch und im Plauderton, aber trotzdem stets wissenschaftlich fundiert.
1 RÜGER, JaN (2017), Helgoland Deutschland, England und ein Felsen in der Nordsee, Berlin, 519 S. Originalausgabe unter dem Titel »Heligoland, Britain, Germany, and the Struggle for the North Sea", Oxford 2017, $370 \mathrm{~S}$.

2 http://catalogue.libraries.london.ac. uk/record=b2683697 S6 (zuletzt abgerufen am 10.5.2018).

3 Es gibt fast vollständige Teilbestände im Landesarchiv Schleswig-Holstein in Schleswig, aber natürlich ohne die handschriftlichen Entwürfe. Der Bestand der Gemeindebibliothek Helgoland (den die älteren hier zitierten Werke wohl zur Grundlage hatten) wurde im 2. Weltkrieg am 18. April 1945 zerstört. Das Exemplar in London ist das einzige vollständige Exemplar.

4 Heute Partnerin in der Partnerschaftsgesellschaft Rechtsanwälte Günther, Hamburg. Ich danke Frau
John für die Durchsicht dieses Beitrags.

5 Ausschnitt aus dem Klappentext. 
Dafür hat er eine eindrucksvolle Zahl von Archiven besucht und eine noch eindrucksvollere Zahl von Texten ausgewertet; allein die Bibliographie ${ }^{6}$ umfasst mehr als 40 Seiten. Auch ausgewiesene Kenner der helgoländischen Geschichte werden in dieser wahren Fundgrube eine Fülle bis dato unbekannter Informationen finden. Rüger breitet ein weites Panorama der verschiedensten Gebiete aus, von den Befindlichkeiten der Gouverneure und der einheimischen Bevölkerung, Fragen der frühen Wirtschaftsförderung, den deutschen Patrioten im Exil, Schmuggel und Badebetrieb, illustren und weniger berühmten Besuchern, Heuschnupfenpatienten, Einführung von deutschen Briefmarken mit dem Konterfei der Königin Victoria bis hin zu Flottenfragen, alles sehr gut belegt und vorbildlich erschlossen: narrative Geschichtsschreibung im besten Sinne.

Es gibt aber doch einen Wermutstropfen: Bei Rüger kommen die Rechtsfragen leider recht kurz und offensichtlich waren ihm die Ordinances nicht bekannt oder trotz seines Archivbesuchs in Schleswig nicht so wichtig. Dabei ist die Darstellung der Geschichte einer Kolonie natürlich ohne das in ihr erlassene und geltende autochtone oder koloniale Recht immer unvollständig. Rüger hat zwar gut erkannt, dass es an der Schnittstelle von gleich fünf Rechtsordnungen (dänisches Recht, englisches (Kolonial-)Recht, schleswig-holsteinisches und später deutsches Recht, nicht-kodifiziertes angeblich "friesisches« Gewohnheitsrecht) eine Fülle von Problemen gab, aber er widmet diesem Thema nur wenige Sätze, obwohl eine eingehendere Beschäftigung mit dieser faszinierenden Problematik sein Buch sehr gut ergänzt hätte.
Die Zeit von der englischen Besetzung $1807^{7}$ bis zum Erlass der ersten Ordinances im Jahr 1864

Am 5. September 1807 nahm Vizeadmiral Thomas McNamara Russell, Oberkommandierender des britischen Nordseegeschwaders, Helgoland vom bisherigen dänischen Gouverneur ohne Gegenwehr ${ }^{8}$ in Besitz. Dänemark, damals eine nennenswerte Seemacht, war mit dem napoleonischen Frankreich verbündet, was auch dazu führte, dass die englische Flotte Kopenhagen ebenfalls im September 1807 in Schutt und Asche legte. In den "Articles of Capitulation " 9 musste Russell den Helgoländern dieselben Privilegien garantieren, die sie schon unter der dänischen Krone genossen hatten. Rüger schreibt dazu: »Es war für die Helgoländer ein großer Vorteil, dass der Kapitulationsvertrag diese Privilegien nicht näher definierte das Dokument avancierte zur heiligsten Verfassungsurkunde der Inselbewohner, auf die sie sich immer dann beriefen, wenn sie ihre Herrscher zu Zugeständnissen zu bewegen suchten. ${ }^{\mathbf{1 0}}$ Die Besetzung Helgolands sollte ursprünglich nicht von Dauer sein, aber die große Bedeutung, die die Insel in den folgenden Jahren als Flottenstützpunkt während der Kontinentalsperre gewann, veranlasste die Engländer, in Artikel 3 des Kieler Friedens vom 14. Januar $1814^{\mathbf{1 1}}$ ausschließlich Helgoland von der Rückgabe der dänischen Territorien auszunehmen.

Helgoland wurde zu einer Kronkolonie, in der dem König grundsätzlich das durch einen Gouverneur ausgeübte Gesetzgebungsrecht zustand. Petersen schreibt dazu: »In den ersten fünf Jahrzehn-
6 In den hier nachfolgenden Fußnoten werden die in Rügers Bibliographie enthaltenen Werke mit allen bibliographischen Angaben zitiert, die Werke aus der hier angehängten Bibliographie in Kurzfassung.

7 Zum Rechtszustand der Insel Helgoland vor 1807 sehr gut und ausführlich Petersen (1938) 1-9. Ein auch angesichts der Entstehungszeit gegenüber den Engländern fairer und sehr kenntnisreicher Artikel.

8 Es gab aber auch nur eine kleine dänische Garnison, »deren Besatzung größtenteils aus Deserteuren und anderen Militärverbrechern bestand, für die der Fels als Verbannungsort galt«, s. Stocks, Heinrich (1927),
Helgoland im Versailler Friedensvertrag, Diss. Greifswald, 11.

9 Text in Weber, Erwin (1986), Beiträge zur Geschichte der Insel Helgoland - aus den Handschriften zusammengestellt, Cuxhaven, 32 (Übersetzung durch den Inselpastor Ingversen als offizieller »interpretor«). Dieser Übergabevertrag wurde anscheinend niemals von der Regierung in London ratifiziert, s.

Moeller, Ernst v. (1904), Die Rechtsgeschichte der Insel Helgoland, Weimar, 248.

10 RüGER (Fn. 1) 26. Ausführlicher zu dieser Widerborstigkeit der Helgoländer (»Widerstand « wäre ein zu expliziter Begriff) die Ausführungen bei
Petersen (1938) 10 ff. Die »Beiträge zur Geschichte der Insel Helgoland « (Fn. 9) enthalten im Original eine Fülle von Eingaben und Beschwerdeschriften Helgoländer Bürger und Institutionen beim Gouverneur und bei der englischen Regierung.

11 Der Kieler Frieden hatte nach der Niederlage Dänemarks in den Napoleonischen Kriegen vor allem die Neuordnung Nordeuropas zum Ziel; die Bestimmungen über die Zukunft Helgolands im dänisch-englischen Teil des Friedensvertrages sind eher als Nebenprodukt zu betrachten. 
ten enthielt die englische Kolonialverwaltung sich jeden grundsätzlichen Eingriffs in die autonomen Einrichtungen Helgolands. “ ${ }^{\mathbf{2}}$ Das lag daran, dass mit der Erwerbung einer Kronkolonie trotzdem das bisher dort geltende Recht fortgelten sollte ${ }^{\mathbf{1 3}}$ und in der Tat galt de facto nicht nur das im Jahre 1807 geltende Recht weiter, sondern es wurden auch danach erlassene schleswig-holsteinische Vorschriften angewendet und noch im Jahre 1890 als rezipiert angesehen. ${ }^{\mathbf{1 4}}$ Petersen berichtet anhand von (Gerichts-)Protokollen, wie es auf der Insel trotz aller Bemühungen der Gouverneure, nicht zuletzt wegen der unterschiedlichen Auffassungen über die anwendbaren Gesetze, zu einer »zunehmenden Verwilderung der Sitten ${ }^{15}$ kam. Es "zeigte sich deutlich, dass die britische Herrschaft in den ersten 50 Jahren offensichtlich äußerst liberal gehandhabt wurde und deshalb zu allerlei Widersetzlichkeiten führte. "16 Als die Bewohner dem Gouverneur Sir Henry King im Jahr 1840 zu seinem 25-jährigen Amtsjubiläum auf der Insel gratulierten, sprach er in seiner Antwort von den »vielen Wohltaten, die die Liberalität Englands der Insel hat angedeihen lassen. Dies sollte die Bewohner eigentlich zur Respektierung ihrer eigenen Gesetze, die ihnen von dem mächtigen Protektor garantiert worden sind, führen. Aber ich habe mit großer Sorge beobachtet, dass diejenigen, die die ersten sein sollten, sie hochzuhalten, viele Gelegenheiten ausgenutzt haben, um sie zu missachten. " ${ }^{17}$ Während die Insel in den Anfangsjahren während der Kontinentalsperre aber noch florierte (es gab sogar eine eigene Handelskammer) ${ }^{\mathbf{1 8}}$ und die Spielbank viele Besucher anzog, ${ }^{19}$ geriet sie in den folgenden Jahren durch schlechtes Wirtschaften, fehlende Verwaltungsstrukturen ${ }^{20}$ und eine unzureichende wirtschaftliche Basis ${ }^{21}$ trotz der Einnahmen aus dem Badebetrieb in eine Schieflage. »Noch unerträglicher wurden diese Verhältnisse, als der Gouverneur Hindmarsh im Jahre 1857 durch Sir Richard Pattinson ${ }^{22}$ ersetzt wurde, dessen Schwäche gegenüber alkoholischen Getränken auch der Öffentlichkeit nicht verborgen blieb. Selbst die äußeren Formen, die bis dahin gegenüber dem Gouverneur als dem Vertreter der englischen Königin immer noch gewahrt waren, gerieten nun ins Wanken. Jetzt endlich setzte sich die englische Regierung ebenso energisch durch, wie sie sich bis dahin passiv verhalten hatte, indem sie - um die Mitte des Jahres 1863 - in der Person das Kapitäns der Garde Maxse ${ }^{23}$ einen vielseitig gebildeten und tatkräftigen Gouverneur ${ }^{24}$ bestellte und mit dessen Hilfe von sich aus eine verfassungsmäßige Neuregelung durchführte. ${ }^{25}$ Und das führt zum Thema dieses Beitrags:
12 Petersen (1938) 25.

13 JANSSEN (2000) $57 \mathrm{f}$.

14 Im Einzelnen erfasst in der amtlichen Übersicht über die Rechtsverhältnisse Helgolands, 1890.

15 Petersen (1938) 29.

16 Oswald Hauser, Vorwort, in: Weber (Fn. 9) 3.

17 Hauser, ebd., englischer Originaltext, in: WeBER (Fn. 9) 57.

18 RüGER (Fn. 1) 47-49.

19 Helgoland war der einzige Ort im Empire, in dem das Glücksspiel - der Steuereinnahmen wegen - geduldet wurde, s. RüGER (Fn. 1) 79 f., 105. Allerdings hat er übersehen, dass mit der Ordinance 1871/1 (»suppression of gaming tables«) das Glücksspiel schlussendlich doch verboten wurde.

20 Es fehlten Polizei- und Vollstreckungsbeamte, so dass Arreste und Landesverweisungen nicht durchgeführt werden konnten, s. Petersen (1938) $28 \mathrm{f}$.

21 Die einzelnen Gründe für den Niedergang (gesunkener Fischabsatz, eingeschränkter Schmuggel, Zurückdrängung des Lotsenwesens) werden im Einzelnen beschrieben bei Sтоскs (Fn. 8) 13.

22 »Komplette Fehlbesetzung«, RüGER (Fn. 1) 100.

$23 \mathrm{Zu}$ Henry Fitzharding Berkeley Maxse, dem einzigen perfekt deutsch sprechenden Gouverneur, den die Insel hatte (er schrieb sogar Theaterstücke in deutscher Sprache zur Aufführung auf Helgoland - das Landesarchiv in Schleswig bewahrt den Text seines Zweiakters »Der Sieg des Herrn Taube «), s. Rüger (Fn. 1) 100-103. S. auch Petersen, Lorenz (1939), Zur Geschichte der Verfassung und Verwaltung auf Helgoland, in: Zeitschrift für schleswig-holsteinische Geschichte $138 \mathrm{f}$. »Sources credit Maxse with a winning personality, a trait all the more important in a small colony where personal contact mattered more that official announcements«, SEIBERT (2013) 202.
24 »Never since Heligoland became a British possession, in 1807 , has its Government been so satisfactorily carried on as during the last ten years. Before 1864 all sorts of abuses were rife, and the morality of the island was at a low ebb, owing the state of Government which then existed ... The Lieutenant-Governor, naturally ignorant of the ancient laws and customs of the island, (was) prepared to allow the inhabitants the greatest liberty«, Heligoland (1874) 576.

25 Petersen (1938) 30. 


\section{Die Ordinances of Heligoland ${ }^{\mathbf{2 6}}$}

Mit dem Datum des 7. Januar 1864 trat nach vorherigen Diskussionen von Maxse mit dem Kolonialministerium $^{27}$ eine ${ }$ Order in Council ${ }^{28}$ making provision for the Government of Heligoland, and Declaring the Powers of the Governor thereof « nach Zustimmung der Königin »by and with the advice of Her Privy Council« in Kraft. ${ }^{29}$

Die Präambel der Verfassung legt nach den oben beschriebenen Zuständen auf der Insel den Grund für deren Erlass fest: »The form of Government now existing in The Island of Heligoland hath been found insufficient for the exigencies of Her Majesty's Subjects resident therein ...«

Die Verfassung enthielt erstmals eine Regelung über die Ernennung eines Gouverneurs ${ }^{30}$ und seine Befugnisse, darunter in Artikel IV das Recht zur Gesetzgebung. Am 6. Februar 1864 wurden daraufhin sehr detaillierte »Instructions « ${ }^{\mathbf{3 1}}$ für den Gouverneur Maxse erlassen, und in diesen findet sich in Art. XVIII folgende Bestimmung: »And We do further direct that all Laws to be made by you shall henceforth be styled `Ordinances made by the Governor of Heligoland .... and that all such Ordinances be drawn up in a simple and compendious form, avoiding, as far as may be, all prolixity and tautology. ${ }^{32}$

Die Weisungen für den Gouverneur enthalten in Art. XXIV folgende Regelung: "And We do further direct that when any Ordinance shall have been made by you, the same shall forthwith be laid before Us for Our final assent, disallowance, or other direction thereupon. ${ }^{33}$

Art. XXXI der Weisungen verbot dem Gouverneur, ohne ausdrückliche Zustimmung der Königin die Insel zu verlassen. Angesichts der negativen Stimmung unter der Bevölkerung war dieses Insel-
26 Die zitierten englischen Originaltexte sind, soweit nicht anders erwähnt, sämtlich in der eingangs erwähnten Sammlung des Institute of Advanced Legal Studies enthalten, die nicht vollständig vorhandenen deutschen Übersetzungen stammen aus dem Archiv in Schleswig. Da sie mit der gleichen optischen Gestaltung und dem gleichen Schrifttyp wie die Ordinances gedruckt wurden, muss es sich um offizielle Versionen handeln. In der Literatur sind keine Angaben über diese Übersetzungen zu finden, auch die Ordinances selbst enthalten keine Bestimmungen über Inhalt, Form und Anwendbarkeit der Übersetzungen. Es steht auch nicht fest, ob alle Ordinances übersetzt wurden oder ob es andere Gründe für die Lückenhaftigkeit gibt.

27 Die Sammlung des IALS enthält eine draft version der Order in Council. Rüger schreibt zum Zustandekommen der Order in Council nach Auswertung des Schriftverkehrs in den Akten der National Archives: „Der eigentliche Beweggrund für das $\mathrm{Zu}$ geständnis einer Verfassung war indes, hier wie fast überall sonst, ein schlichter Tauschhandel: politische Freiheit gegen finanzielle Eigenverantwortung ... Man hielt die Inselbewohner für 'primitiv und sungebildet , aber sie waren weiße Europäer und hatten damit Anspruch auf
Gleichbehandlung mit den britischen Siedlerkolonien".

28 Von den deutschen Autoren (Petersen, Moeller etc.) ॥Verfassung « genannt, dieser Begriff wird von Rüger in seiner Beschreibung (102-106) übernommen - eine Bezeichnung als "Grundordnung« wäre wohl angemessener, nicht zuletzt, weil nach ihrem Art. XXIII diese Order nur eine Geltungsdauer von fünf Jahren haben sollte. Petersen nutzt einmal (30) den passenden Begriff der »oktroyierten Verfassung «. In diesem Text wird trotzdem das Wort »Verfassung" gebraucht, weil es sich im Schrifttum durchgesetzt hat.

29 Vollständiger Text in Weber (Fn. 9) $75 \mathrm{f}$.

30 »Die Befugnisse des Helgoländer Gouverneurs sind vor dem Jahre 1864 nicht gesetzlich festgelegt worden. Es steht nicht einmal fest, ob diesen Beamten bei ihrer Ernennung auch nur eingehende Dienstinstruktionen mit auf den Weg gegeben worden sind ", Moeller (Fn. 9) 249.

31 Ebenfalls in der Sammlung des IALS enthalten, deutsche Fassung in Weber (Fn. 9) 78.

32 Eine wunderschöne Formulierung, die sich als Maßstab für heutige Normgebung auch gut machen würde; deutsche Übersetzung: »....und müssen alle Verordnungen kurz und klar gefasst sein, jede Weitschweifig- keit oder Wiederholung so viel wie möglich vermeidend «, WeBER (Fn. 9) 80.

33 Die Fassung des IALS enthält in der Regel den handschriftlichen Entwurf und die gedruckte Version der Ordinances. In keinem Fall gibt es Abweichungen der Druckversionen von den Entwürfen, so dass die Anfertigung der Druckversion wohl jeweils als royal assent zu deuten ist. Zur kolonialen Gesetzgebung näher: Commonwealth Enactments $\left(1952^{3}\right)$. Zwischen den datierten handschriftlichen Entwürfen und den datierten Druckversionen lag jeweils ein Zeitraum von sechs bis acht Wochen, was sicher teilweise auf die Prüfung im Kolonialministerium und teilweise auf den Postweg zurückzuführen ist. In der Literatur waren keine Hinweise zu finden, wie der jeweilige Postverkehr vom Gouverneur zum Kolonialministerium und zurück tatsächlich und praktisch erfolgt ist. Aus der Ordinance 1867/1 ergibt sich aber, dass auf der Insel ein hamburgisches Postamt bestand, das im Jahre 1867 an die englische Regierung übertragen wurde. Es ist nicht ganz auszuschließen, dass die Kolonialverwaltung bis 1867 für ihren Postverkehr keinen ausländischen Postdienst nutzen durfte. 
leben mit allen damit verbundenen gesellschaftlichen Einschränkungen ${ }^{34}$ wohl nur für einen starken Charakter wie Maxse zu ertragen. Denn die Verfassung von 1864, die den Helgoländern viele Mitbestimmungsrechte einräumte (bzw. einräumen wollte), allerdings unter Aufsicht und Anleitung des Gouverneurs, scheiterte an »Trotz und Rechthaberei der Helgoländer «. ${ }^{35}$ Die »Beiträge ${ }^{\mathbf{3 6}}$ enthalten eine von 232 Helgoländern (und 77 Helgoländer Witwen) unterzeichnete und persönlich von einem Bürgerkomitee in London übergebene »Beschwerdeschrift über den Gouverneur Maxse " an die englische Regierung, mit der diese verlangten, »dass den Bürgern ihre alten Rechte, die England ihnen garantirte (sic), wieder gegeben und das alte Recht und Gesetz wieder in Kraft gesetzt werde «. ${ }^{37}$ Die Lage war dergestalt, dass der Kolonialstaatssekretär, der Herzog von Buckingham, sich 1867 persönlich an Bord eines Kriegsschiffes nach Helgoland begab, um den Helgoländern eine Änderung der Verfassung von 1864 in Aussicht zu stellen. ${ }^{38}$ Aber der Gouverneur und der Herzog »sollten schwer enttäuscht werden «. 39 Nachdem die Helgoländer auch noch in einen Steuerstreik getreten waren und sich die Gemeindevertretung außerstande sah, einen Vorsitzenden zu wählen ${ }^{\mathbf{4 0}}$, erklärte der town clerk Michels »es öffentlich als seine entschiedene Meinung, die Helgoländer seien für eine Volksvertretung nicht geeignet, unter den von ihnen gewählten Vertretern seien nur private Beziehungen und persönliche Interessen ausschlaggebend $\ll .{ }^{41}$ Daraufhin gab die Regierung in London am 29. Februar 1868 eine neue Order in Council ${ }^{42}$ bekannt. So lang und kompliziert ${ }^{43}$ die Verfassung von 1864 auch war, die neue Verfassung enthielt nur noch einfache Regelungen: Der Gouverneur wurde jetzt ohne jede Einschränkung zum Erlass aller Gesetze ermächtigt und das einzige Organ, das jetzt noch beratend tätig war, war ein executive council, dessen Mitglieder er selbst auswählte und ernannte. ${ }^{\mathbf{4 4}}$ "The second constitution virtually abolished Helgolandian self-governance, but restored order. « ${ }^{45}$

Die nachfolgend aufgeführten und zum Teil auch erläuterten Ordinances von der ersten aus dem Jahr 1864 bis zur sechsundsiebzigsten im Jahr 1889 zeigen sehr gut, dass sie mit wenigen Ausnahmen geschaffen wurden, um die Probleme Helgolands als einer kleinen, wirtschaftsschwachen und geographisch isolierten Insel in den Griff zu bekommen.

Die erste Ordinance vom 2. Mai 1864 »for the better administration of justice« bzw. »für bessere Rechtspflege daselbst« sah die Einführung einer dreistufigen Gerichtsbarkeit (Police Court, Court of Sessions, Court of Appeal) nach dem Jurysystem vor. Wegen des anhaltenden Widerstands der Inselbevölkerung gegen die Einführung eines Gerichtssystems nach englischem Vorbild scheiterte die Umsetzung, so dass die Ordinance nicht zur Anwendung $\mathrm{kam}^{46}$ und nach der Einführung der Verfassung von 1868 durch die Ordinance 1868/3 ersetzt wurde. Die Insel mit damals nur einigen Hundert Bewohnern war aber auch vermutlich zu klein und mit der Einführung einer dreistufigen Gerichtsbarkeit nach dem Vorbild anderer Kolonien überfordert. Ebenfalls vom 2. Mai 1864 stammen die zweite und dritte Ordinance über die Bestellung von zwei Quartermasters (je ein Beamter für das Unter- und Oberland mit Aufgaben für Verwaltung und Vollstreckung, 1864/2) und das Wahlverfahren für die Gerichte (1864/3).

Die Ordinances 1864/4 und 1864/5 beschäftigen sich mit der Einstellung ${ }^{47}$ und Bezahlung ${ }^{48}$
34 Lady Maxse war die österreichische Hofschauspielerin Augusta von Rudloff.

35 Moeller (Fn. 9) 249.

36 WeBer (Fn. 9) $86 \mathrm{ff}$.

37 Ebd., 94.

38 Petersen (Fn. 23) 147; Ritsema (2007) 81.

39 Petersen, ebd.

40 Ebd., 148.

41 Ebd., 148 f. mit Nachweisen.

42 In der Sammlung des Foreign Office nicht enthalten, englische Originalversion in Weber (Fn. 9) $110 \mathrm{f}$.

43 »Extremely complex«, Ritsema (2007) 81.
44 Heligoland (1874) $577 \mathrm{f}$.

45 Seibert (2013) 202.

46 Gilt auch für die Ordinance 1865/1 über Geschworenengerichte und 1865/4 über das Wahlverfahren.

47 An der Einstellung dieser Mitarbeiter entzündete sich unter anderem wegen der damit verbundenen Kosten der Zorn der Helgoländer.

48 Die Bezahlung wie überhaupt die gesamte Haushaltführung der Insel erfolgten bis 1871 nicht in Pfund Sterling, sondern nach der in Norddeutschland weitverbreiteten hamburgischen Courantmark; nach der Reichsgründung in Reichsmark. Da- nach erhielt ein Lehrer zweiter Klasse 125 Mark jährlich, ein Lehrer dritter Klasse 100 Mark jährlich. Weitere Regelungen zum Thema Bezahlung sind nach den ersten Ordinances direkt in den jeweiligen Ordnungen enthalten. 
weiterer Mitarbeiter und mit Schulfragen, ${ }^{49}$ die folgende Ordinance 1864/6 mit der "gütlichen Ausgleichung von Lotsensachen und Streitigkeiten ähnlicher Art«.

Von allen Ordinances zeigt die Nummer 1864/7 am besten, dass sich die englischen Kolonialbehörden nach Kräften bemühten, den Befindlichkeiten der Inselbewohner entgegen zu kommen. Es ist die Ordinance for establishing the Law Code of Heligoland, die folgendes bestimmt: "Das letzte bis zum Jahr 1864 erlassene Schleswig-Holsteinische Civil- und Kriminalgesetz soll als Grundlage dienen für vom Gesetzgebenden Rat ${ }^{50}$ von Helgoland $\mathrm{zu}$ erlassende Gesetze.« Jedoch: »Diese Anordnung macht der Rücksicht der Engländer auf die deutsche Stammesart der Helgoländer alle Ehre. Aber sie wirkt dadurch geradezu komisch, dass jenes mythische Zivil- und Kriminalgesetzbuch Schleswig-Holsteins überhaupt nicht existierte ... Ohne Zweifel handelte es sich lediglich um Irrtum und Unkenntnis der englischen Behörden. ${ }^{51,52}$

Der ungewöhnlich detaillierte Entwurf für die Einführung eines Wreck Court (nach der Zählung 1864/8), der sich mit den für Helgoland enorm wichtigen Rechten und Streitigkeiten an Strandgut befasste, trat nicht in Kraft, an seiner Stelle wurde die Ordinance 1865/12 erlassen. Die Gründe dafür sind aus den Akten nicht ersichtlich. Auch die späteren Verordnungen zum Strandrecht ${ }^{53}$ zeigen, wie wichtig das Thema für die Helgoländer war und wie sehr der Gouverneur versuchte, massive Missbräuche zu beschränken.

Eine Art frühe Umweltschutzregelung (aber mit wirtschaftlichem Hintergrund) war die Verordnung 1865/3 »enacting that the fishing of the Heligoland Oyster Bank shall be conducted by the Colonial Government« mit folgender Begründung: »Die Befischung der Helgoländer Austernbank wurde von den Einwohnern der Insel im höchsten Grade vernachlässigt und führte in finanzieller Hinsicht zu großem Schaden. ${ }^{\mathbf{5 4}}$ Diese Verordnung enthält präzise Regelungen über den Schutz und die wirtschaftliche Nutzung der Austern.

Die Verordnungen 1865/5, 1868/2 und 1884/1 beschäftigen sich mit einem damals sehr bedrückenden Thema: der Vormundschaft und der Vermögensverwaltung für Witwen und Waisen. Da viele Helgoländer sich mangels anderer Erwerbsmöglichkeiten als Seefahrer verdingen mussten, kam es in diesem Personenkreis häufig zu Todesfällen und es bedurfte eines nach damaligen Standards wirksamen Vermögensschutzes ihrer Angehörigen. Diese beiden Verordnungen enthielten die wichtigsten Veränderungen des ansonsten weitgehend weitergeltenden materiellen Rechts.

Mit den auf der Insel stets besonders umstrittenen Steuern, Abgaben und der Vergabe von Lizenzen beschäftigt sich ab 1865 eine ganze Reihe von Ordinances, beginnend mit Ordinance 1865/6: $1865 / 7,1865 / 8,1865 / 9,1865 / 10,1868 / 1,1868 / 5$, $1872 / 1,1878 / 1,1879 / 1,1879 / 2,1880 / 1,1882 / 2$, 1883/2, 1884/2, 1884/3, 1884/4, 1888/1: die Kolonialverwaltung brauchte Geld! Und sie beschaffte sich dies mit Einkommensteuern, Gewerbesteuern, Jagd- und Hundesteuern, Kurtaxen, Einfuhrzöllen und den Überschüssen aus dem Postdienst.

In den unruhigen Jahren 1866 und 1867 gab es jeweils nur eine Ordinance über die Anstellung von Kirchenvorstehern und über die Postverwaltung der Insel, modifiziert durch Ordinance $1886 / 2$.

In der Sammlung des IALS befindet sich allerdings ein handschriftlicher Entwurf mit der Ordnungsnummer 1866/2 über eine erneute Verbesserung der Arbeit der Gerichte (»for the better administration of justice«), an dem eine Fülle von Korrekturen angebracht wurde, insgesamt sind fünf verschiedene Handschriften identifizier-
49 Dazu auch die Ordinances 1865/2 und 1868/6 (Schulpflicht vom 6. bis zum 14. Lebensjahr); Zeitdokumente mit sehr präzisen Vorgaben über die Unterrichtsinhalte und die Umstände des Unterrichts: »Für die Heizung der Schulzimmer hat jeder Schüler täglich ein Stück Torf mitzubringen. Verboten ist ein halbes oder viertel Stück Torf« (1865/2, Art. XLV).

50 Wie oben beschrieben: mit der Verfassung von 1868 aufgelöst.
51 Moeller (Fn. 9) 246.

52 »Der letzte englische Gouverneur Barkly, dem die Zusammenhänge nicht bekannt waren, forderte noch im Jahre 1889 ... das in jener Ordinance erwähnte Gesetzbuch an und musste sich ... belehren lassen, dass es derartiges nicht gebe«, Petersen (1938) $31 \mathrm{f}$.

53 Ordinances 1865/11, 1868/7, 1876/1. "Not withstanding numerous complaints ... the so-called sStrandrecht continued to hold its own on the island as one of the most cherished of the old rights and privileges. In fact (it) became little better than legalised piracy«, Heligoland (1874) 577.

54 Ähnlich die Ordinance 1887/1 for the Preservation of Sandy-Island (»Düne«). 
bar. Er erlangte vermutlich keine Gesetzeskraft, an seine Stelle trat die oben beschriebene Ordinance 1868/3.

Im Jahr 1869 wurden keine Ordinances erlassen; vermutlich ein Zeichen dafür, dass mit der neuen Verfassung von 1868 etwas Ruhe eingekehrt war - die Ordinances unter der neuen Verfassung waren nun nur noch »enacted by the Governor of Heligoland «.

Im Jahre 1870 wurde eine Ordinance über Testamente erlassen (1870/1) und eine Verordnung über die Inselapotheke (1870/2).

Im Jahr 1871 wurde die Möglichkeit, auf der Insel Glücksspiele anzubieten und durchzuführen, nach wiederholten Mahnungen des Colonial Office abgeschafft (1871/1 mit Strafbestimmungen). Erstaunlicherweise scheint gerade für diese wichtige Ordinance keine deutsche Übersetzung vorzuliegen. 55

Die Ordinance 1871/2 eröffnete die Möglichkeit, außerhalb von Helgoland gemachte Schulden $\mathrm{zu}$ vollstrecken, eine Art frühes internationales Zwangsvollstreckungsrecht (»Ordinance for the better recovery of depts, contracted abroad by Heligolanders or persons residing in Heligoland «). Auch dafür gab es aber dringenden Handlungsbedarf: "Meanwhile, no debts could be recovered in the island; no legal decrees enforced; the credit of the island was at the lowest ebb, and merchants in Hamburgh, Altona, and Bremen knew only too well that the chance of their recovering a disputed debt was about equal to the chance of a ship or cargo being got off the reefs of Heligoland, on which latter point the underwriters considered that in such a case a vessel should be scratched off the books at once without wasting further trouble or expense. ${ }^{56}$

Mit der Ordinance 1872/2 wurde ein Gericht für Scheidungs- und Ehesachen eingesetzt. Das ist nun eine besonders interessante Verordnung, in der die Berechtigten (Personen, die die Ehe auf Helgoland geschlossen haben und von denen wenigstens ein Ehepartner mindestens zwei Jahre auf der Insel wohnhaft war, Artikel IV), Scheidungs- gründe, finanzielle Scheidungsfolgen und auch schon Bestimmungen über "Schutz, Verpflegung oder Erziehung der Kinder" (Art. XIII) enthalten waren (ergänzt durch Ordinance 1884/5).

Die Verordnung 1873/1 (einzige Verordnung des Jahres) ist ganz erstaunlich, aber es ist davon auszugehen, dass es für ihren Erlass Gründe gab, nach der Präambel war sie »expedient«: Verordnung zur Verhinderung böswilliger Beschädigung von Telegraphen, Unterseekabeln, Schiffssignaloder Semaphore-Stationen (mit Strafbestimmungen). Vielleicht war es aber auch nur eine vorsorgliche Maßnahme - auf der Insel wurde sie offensichtlich nicht diskutiert, denn in der umfangreichen Sammlung von Weber (Fn.9) finden sich keine Materialien zum Thema.

Die Sammlung des Colonial Office enthält für das Jahr 1874 ein Blatt mit der schlichten Bemerkung: "No ordinances passed in 1874 «.

Das Jahr 1875 endete mit der Verordnung $1875 / 1$ vom 1. Dezember über »die Registration von Sterbefällen, sowie das rechtzeitige Begräbnis Verstorbener« (aus der angehängten Gebührentabelle ergibt sich, dass damals fünf Pfund Sterling einen Wert von 100 Mark hatten). In $\mathrm{Zu}$ sammenhang damit: die Verordnung 1875/2 vom selben Tag "relating to wills« über die sichere Verwahrung von Testamenten.

Nach mehreren Verordnungen zum Steuer- und Strandrecht (mit der Ordinance 1876/1 wurde das helgoländische Strandrecht an die deutsche Strandungsordnung vom 17. Mai 1874 angepasst) aus den Jahren 1876 bis 1882 (in diese Zeit fällt auch der Wechsel von Gouverneur Maxse auf den vorletzten Gouverneur O'Brien) ist als nächste wichtige Ordinance die Verordnung 1882/3 über die "limitation of actions " (mit unterschiedlichen Verjährungsfristen für die verschiedenen Ansprüche) zu nennen.

Mit der Ordinance 1883/1 (»betreffend den Kleinhandel mit Spirituosen«) beginnt der Versuch, die Probleme zu regulieren, die sich bis heute aus dem Status der Insel als »Fuselfelsen« ergeben; mit derselben Thematik beschäftigen sich auch
$55 »$ Our red sandstone rock off the mouth of the Elbe is no longer a little hell in which German bathing guests may lose money, reputation, and temper ... Heligoland without gaming tables, without wreckers, and with a revenue, is a tribute to the able management of his Excellency Fitz

Maxse, Lieut. Colonel and Gover-

nor«, Heligoland (1872) 641.

56 Heligoland (1874) 577. 
die Ordinances 1883/4, 1883/6, 1884/6 (mit der schönen deutschen Übersetzung: „Verordnung den Verkauf berauschender Getränke an Gewohnheits-Säufer verbietend «), 1885/1 und 1886/3.

Hochinteressant geht es weiter mit der Ordinance $1883 / 3$ : establishing new weights and measures for use of Colony. Mit dieser Ordinance wurde das metrische System (Gewichte, Längenmaße, Hohlmaße) ganz im Gegensatz zum Empire eingeführt. Die modernen Zeiten spiegelten sich dann auch in der Ordinance 1883/5 wider, die die Einrichtung einer Sparkasse (mit durch die Inselkasse garantierten Einlagen, bei 3\% Zinsen) bestimmte.

Wegen der bevorstehenden und sich schon am Horizont abzeichnenden Übergabe Helgolands an Deutschland erlangten die am Ende erlassenen Verordnungen der Kolonialverwaltung 1888/2 (»An Ordinance to establish Quarantine when necessary in Heligoland «), 1888/3 (»An Ordinance to protect Copyright in Paintings, Drawings and Photographs«), 1888/4 (»An ordinance to prevent aliens from acquiring rights in Heligoland by marriage with Heligoland women«), 1888/5 (»An Ordinance to regulate the construction of Buildings«), 1888/6 (Änderungen der Schulverordnung 1868/6), 1888/7 (»An ordinance to make provision for vaccination in Heligoland«), 1889/1 (unter dem letzten Gouverneur Arthur Cecil Stuart Barkly, über den letzten Haushalt der Kolonie in Höhe 156.265,50 Reichsmark), 1889/2 ${ }^{57}$ (»An Ordinance to give power to the Governor to prohibit when necessary the exportation of warlike Articles«), 1889/4 (»An Ordinance to provide for the summary punishment of perjury«), 1889/5 mit Änderungsverordnung 1889/6 (»An Ordinance to make better provision for the protection of women and girls and other purposes«), 1889/7 vom 1 . November 1889 (»An Ordinance to prevent the imposition of fraudulent marks on Merchandise«) keine große Bedeutung mehr.

Es ist nicht ganz verständlich, warum die Kolonialverwaltung bis zuletzt eine so große gesetzgeberische Aktivität entfaltete. Vielleicht wollten die Engländer nur mit modernen Regeln ein Zeichen gegenüber dem kaiserlichen Deutschland setzen, denn nach Art. XII (Abtretung Helgolands) Nr. 4 des Vertrages zwischen Deutschland und England über die Kolonien und Helgoland vom 1. Juli 1890 (»Die zur Zeit bestehenden heimischen Gesetze und Gewohnheiten bleiben soweit es möglich ist, unverändert fortbestehen«) war zu erwarten, dass die letzten Ordinances wenigstens für einen gewissen Zeitraum Bestand haben würden. Es ist anders gekommen, denn das Gesetz über die Vereinigung von Helgoland mit dem Deutschen Reich vom 15. Dezember 1890 sah in seinem $\$ 6$ lediglich Übergangsfristen (»soweit die Schonung der auf der Insel bestehenden Gewohnheiten und Gesetze es erheischt«) vor, die durch die Verordnung über die Einführung von Reichsgesetzen und preußischen Landesgesetzen auf der Insel Helgoland vom 14. Dezember 1892 präzisiert wurden. Mit Ausnahme des Zollwesens war die Übernahme von Reichsrecht und preußischem Recht im Jahre 1910 abgeschlossen. ${ }^{58}$

Es ist überhaupt ganz anders gekommen, als man sich das im Jahre 1890 hätte vorstellen können. Diese Geschichte der Insel bis heute wird von Rüger - mit sehr gelegentlichen kurzen Ausflügen ins Recht, um beim Thema zu bleiben unter einem englisch-deutschen Blickwinkel beschrieben. Er muss aber auch feststellen, dass die Insel seit ihrer erneuten Rückgabe 1952 im Verhältnis der beiden Nationen keine Rolle mehr spielt, so dass sein $»$ Epilog ${ }^{59}$ doch etwas sehr bemüht wirkt.

Chronologische Liste der Ordinances of Heligoland ${ }^{60}$

An Ordinance ...

1864/1: for the better administration of justice 1864/2: for the appointment of quartermasters 1864/3: for regulating the proceedings at the elections for the combined court

1864/4: appointing the salary of the third Stipendiary Magistrate
57 Nach einem handschriftlichen Vermerk in den Akten des Colonial Office gab es keine Ordinance 1889/3.

58 Sтоскs (Fn. 8) 21. Zolltechnisch ist Helgoland bis heute EU-Sondergebiet.
$59371 \mathrm{ff}$.

60 Die Übersicht von Petersen (Fn. 23) 161-163 enthält inoffizielle, von ihm erdachte Kurztitel in Deutsch. 
1864/5: for opening the office of $1^{\text {st }}$ class school teacher to a layman, and for increasing the salaries ...

1864/5: appointing the pilot inspector and four quartermasters

1864/7: for establishing the Law Code of Heligoland

1864/8: enacting rules and regulations for the conducting of the business of the Wreck Court

1865/1: to amend an Ordinance relating to trial by jury

1865/2: enacting rules and regulations for the conduct of the Colonial School

1865/3: fishing of the Heligoland Oyster Bank shall be conducted by the Colonial Government

1865/4: to amend an Ordinance relating to Candidates for election to Combined Court

1865/5: enacting that the Court of Sessions shall take measures for the guardianship of minors ...

1865/6: enacting taxation for ecclesiastical and educational purposes

1865/7: enacting taxation on real and personal property

$1865 / 8$ : enacting that the medical fees due to the Doctor of the island for the time being shall ...

1865/9: establishing a Finance Committee

1865/10: for imposing import duties on Wine and Spirits

1865/11: enacting amendments to the existing strand laws of Heligoland

1865/12: enacting certain rules as regards the conduct of the business of the Wreck-Court

1866/1: relating to the appointment of Churchwardens

1867/1: enacting rules and regulations for the conduct of the Island Post Office

1868/1: enacting taxation on the letting of lodgings to Bathing-guests and visitors of the island ...

1868/2: vesting the property of widows and minors in the hands of trustees

1868/3: for the better administration of justice

1868/4: for imposing import duties on wines, spirits and beer

1868/5: enacting taxation on real and personal property

1868/6: enacting rules and regulations for the conduct of the Colonial School

1868/7: enacting amendments to the existing Strand Laws of Heligoland

1870/1: providing for the better execution of deeds, wills and testamentary dispositions
1870/2: for the better direction of the Apothecary's business in Heligoland

1871/1: for the suppression of Gaming Tables

1871/2: for the better recovery of Debts contracted abroad by Heligolanders or persons residing ...

1872/1: imposing a duty on spirits in cask, according to the strength of such spirits

1872/2: constituting a Court for Divorce and Matrimonial causes

1873/1: for the prevention of malicious injuries to Telegraphs, Submarine Cables, Ship signal ...

1875/1: to provide for the registration of deaths and the timely burial of deceased persons

1875/2: relating to Wills

1876/1: enacting amendments to the Strand Ordinance of Heligoland

1878/1: for imposing import duties on wines, spirits and beer

1879/1: imposing import duties to petroleum

1879/2: enacting the Licenses shall be taken out annually by all Public Houses and other places ... 1880/1: imposing import duties on petroleum 1882/1: to amend an explain ordinance No. 1 of 1878

1882/2: for ensuring the due and punctual payment of taxes

1882/3: for the Limitation of Actions

1883/1: to regulate the sale of spirits by retail

1883/2: to increase the Import duty on Petro-

leum and to provide for the safe keeping of Petroleum

1883/3: establishing new weights and measures for use of Colony

1883/4: to amend Ordinance No. 1 of 1883

1883/5: to establish a Government Savings Bank in Heligoland

1883/6: for licensing retail traders

1884/1: vesting the property of widows and minors in the hands of trustees

1884/2: to provide for the taxation of Visitors to

Heligoland residing in Hotels or Lodginghouses ... 1884/3: to provide for the licensing of Boats and

Watermen and to ensure safety and comfort ...

1884/4: to amend Ordinance No. 1 of 1882

1884/5: to amend ordinance No. 2 of 1872

1884/6: to prohibit the sale of intoxicating liquors to Habitual Drunkards

1885/1: to provide against the sale of unwholesome food, and the Adulteration of food and drugs

1886/1: to prohibit the taking on board of Her Majesty's Ships ... of Spiritous and other liquors 
$1886 / 3:^{61}$ to provide for the preservation of the Public Health

1887/1: to provide for the preservation of Sandy-Island

$1887 / 2$ : with respect to the protection of the Post-Office and to offences committed in relation ...

1888/1: to impose a duty on licenses to use and carry guns

1888/2: to establish Quarantine when necessary in Heligoland

1888/3: to protect Copyright in Paintings, Drawings and Photographs

1888/4: to prevent aliens from acquiring rights in Heligoland by marriage with Heligoland women

1888/5: to regulate the construction of Buildings
1888/6: to amend and explain Ordinance 6 of 1868

1888/7: to make provision for vaccination in Heligoland

1889/1: to apply a sum not exceeding $\mathrm{Rm}$. $156,256.50$ towards the service of the year 1889

$1889 / 2$ : to give power to the Governor to prohibit when necessary the exportation of warlike Articles

$1889 / 4:^{62}$ to provide for the summary punishment of perjury

1889/5: to make better provision for the protection of women and girls and other purposes

1889/6: to amend Ordinance No. 5 of 1889

1889/7: to prevent the imposition of fraudulent marks on Merchandize

\section{Bibliographie}

(enthält nur Werke, die in der Bibliographie von RüGER ${ }^{63}$ nicht berücksichtigt wurden)

- Commonwealth Enactments $\left(1952^{3}\right)$, in: Halsbury's Laws of England, Vol. 15, 668

- Degn, Christian (1994), Schleswig-Holstein - eine Landesgeschichte, Neumünster

- Denkschrift über die Beweggründe zu dem deutsch-englischen Abkommen. Erste Beilage zum Deutschen Reichsanzeiger und Königlich preußischen Staatsanzeiger, Nr. 181, Berlin, 29.07.1890

- Dupont, Jerry (2001), Heligoland, in: Ders., The Common Law Abroad - Constitutional and Legal Legacy of the British Empire, Littleton, 1144-1148

- Gottrried, Leo (1927), Die Erwerbung Helgolands durch Deutschland, Diss. Wien

- Heligoland (1872/74), in: Nautical Magazine 641, 576-579

- Нӧсн, Тномаs (1995), Der Erwerb Helgolands 1890, in: Der Einigungsvertrag zwischen völkerrechtlichem Vertrag und nationalen Gesetz, Berlin, 61-64

- Janssen, Helmut (2000), Die Übertragung von Rechtsvorstellungen auf fremde Kulturen am Beispiel des englischen Kolonialrechts, Tübingen

- Klimpel, Jürgen (1965), Die neuzeitliche Entwicklung der Inselgemeinde Helgoland, Konstanz

- Krüss, Erich-Nummel (2013), Chronologie der Insel Helgoland, 7. Aufl., Helgoland

- Lachmann, Günter (1990), 1890 - Das Helgoland-Sansibar-Abkommen. Vorgeschichte, Inhalt und Wirkungen des deutschenglischen Vertrages, in: Helgoland - 100 Jahre deutsch, hg. von der Gemeinde Helgoland, Otterndorf, 17-21

- Mueller, Christian, Henry Peter Rickmers (2000), Auf Helgoland ist alles anders - die wechselvolle Geschichte einer europäischen Insel, Hamburg

- OetKer, Friedrich (1878), Verfassung und Recht auf Helgoland, Stuttgart

- Petersen, Lorenz (1937/38), Aus dem Helgoländer Rechtsleben, Teile I bis III, in: Schleswig-Holsteinische Anzeigen vom 15.04.1937, 01.01.1938 und 01.02.1938

- Ritsema, Alex (2007), Heligoland, Past and Present, London

- Rothfels, Hans (1924), Bismarcks englische Bündnispolitik, Stuttgart

- Seibert, Sebastian (2013), Heligoland under British Rule 1807-1890, in: Matthews, Jodie, Daniel Travers (Hg.), Islands and Britishness. A Global Perspective. Newcastle, 193-206

- Übersicht über die Verwaltungseinrichtungen und Rechtsverhältnisse Helgolands, in: Stenographische Berichte über die Verhandlungen des Reichstages, Zweiter Anlageband Nr. 145, 1891, 923 ff.

61 Ordinance 1886/2 wurde nicht gedruckt zurückgezogen und gleich durch Ordinance 1887/2 ersetzt.
62 Ordinance 1889/3 wurde vor Veröffentlichung zurückgezogen.

63 RüGER (Fn. 1) 468-507. 